\title{
INTERNET OF THINGS BASED MODEL FOR IdENTIFYing Pediatric EMERgency CASES
}

\author{
Juliet Gathoni Muchori ${ }^{1}$, Gabriel Kamau ${ }^{1}$, and Faith Mueni Musyoka ${ }^{2}$ \\ ${ }^{1}$ Department of Information Technology, Murang'a University of Technology, \\ Murang'a, Kenya \\ ${ }^{2}$ Department of Mathematics, Computing \& Information Technology, University of \\ Embu, Embu, Kenya
}

\begin{abstract}
Pediatric emergency cases need rapid systems that measure vital body parameters data, analyze and categorize emergency cases for precise action. Current systems use manual examination resulting in delayed medication, death, or other severe medical conditions.In this paper, we propose a Internet of Things (IoT) based model, created using Balena fin with Raspberry pi compute module. It is used for determining emergency cases, in pediatric section, specifically the triage section. It is later tested using hospital data that represents the vital parameters in pediatric. Our approach entails designing and setting up the hardware and software infrastructure, to accommodate data via Bluetooth protocol, and transmit it to the cloud server database via Message Queuing Telemetry Transport (MQTT). Later, we perform machine learning on the data by training a model and finally develop a Plotly Dash analytical application integrating the model for visualization near real-time.Findings show that emergency cases are detected using vital body parameters which include the body temperature, oxygen levels, heart rate and the age. The model indicates a 97\% accuracy.In conclusion, children's emergency cases are detected in time using IoT gadgets and machine learning classification.
\end{abstract}

\section{KEYWORDS}

Internet of Things, message queuing telemetry transport, Amazon web service, World health organization

\section{INTRODUCTION}

Children's health emergency cases are on the rise. The need for convenient, efficient, affordable, urgent, and preventive medication has led to the development of e-health models over the past years. These models measure vital body parameters, analyze and diagnose diseases and conditions to aid medical decision-making. Current systems are manual therefore, they need specialization and automation to enhance service delivery in hospitals.

Modern e-health models are based on cloud computing, IoT gadgets, wearable sensors, and modern data analytical methods. Some are also linked to medical databases for efficiency and data retrieval.

The United Nations Convention on the Rights of the Child (UNCRC) Lansdown stated that children have a right to be heard and participate in healthcare issues [1]. Among the issues include understanding their health conditions, giving their views, and participating in health decision-making. Currently, parents and healthcare professionals are the ones who take that role. Usage of e-health solutions can remove the barriers and assist children to communicate with health care professionals [2]. The use of IoT gadgets will assist the children to express themselves electronically by analyzing their vital body parameters and hence emergency status. 
Advanced Medical Sciences: An International Journal (AMS) Vol 8, No.1/2/3, August 2021

In this paper, we propose an IoT based model, that employs peripherals and BalenaFin gadgets with Raspberry Pi compute module at its core for vital body parameter data collection. The gadgets use Bluetooth and other protocols such as MQTT for data communication. Data is sent to a cloud server database infrastructure and later machine learning techniques are applied to the data to categorize children into emergency, no emergency, and moderate emergency.

Several models on e-health have been proposed showing that there are severalIoT gadgets made by different manufacturers for semantic sensing [3]. These devices collect data using different data formats, which causes a problem in device interoperability, and data normalization. Jin and Kim [3] created an e-health model that addresses interoperability issues in the IoT devices and supporting data with different style formats.

The rest of the paper presents related works methodology used, implementation, results, discussion, and conclusion.

\section{RELATED WORKS}

Studies focusing on children cases exist, for instance, Jangra and Gupta created a system for monitoring and recording patients data [5]. The model consisted of three sensors that collected heart rate, temperature, and blood pressure data from the patients, raising alerts whenever it encountered abnormalities. In [5] normal Blood Pressure range was $80-120 \mathrm{~mm} \mathrm{Hg}$ while the body temperature normal range was 36.5-37.5C and Heart Rate normal range was 60-100 beats/min. The model also used the data collected to analyze and predict chronic disorders using data mining techniques. The challenge with the model is that it is suited for specific chronic diseases such as heart attack.

In [3], a model is developed to address the device interoperability issue and normalization of data. It proposes various gadgets that measure different parameters, for instance, the IoT blood pressure gadget is used to measure blood pressure-related data, electromyography gadgets collect data related to body muscles, and the galvanic skin response gadget is used to gather patients' emotional behaviors. Further, a close look at these technologies shows that most analysis of a patient's information involves temperature, blood pressure, muscle data, and skin responses. However, the challenge with the model is that it only tested interoperability issues and largely neglected data analysis.

Another related low-cost sensor e-health model that offers medical services was also created [6]. The model uses low power and has an increased data accuracy [6]. It can perform medical checks using Sensor Controllers (SC), and the results are communicated to mobile or tablet devices, from SC and logical gateways. The model, which uses IoT gadgets like body position sensor, ECG, Airflow, electromyogram-EMG, thermometer, glucose sensor, galvanic skin response sensor, and blood pressure gadgets, has been shown to produce accurate results hence good for hospitals. The limitation of the model is that it fails to indicate the level of emergency of a patient.

Another related model constructed using different IoT gadgets which assisted in collecting data about the quality of air, the temperature, detection of earthquakes, level of light, level of humidity among others is proposed [4]. Using this data, the model makes meaningful insights for decisionmaking. IoT gadgets used in water management were also used to ensure the water is well distributed within the hospital premise efficiently. The gadgets assisted to reduce the wastage of water via leaking. The IoT gadgets were used to monitor when the dustbin was full and needed to be emptied. This reduced the human efforts to keep on checking the bin now and then in addition 
Advanced Medical Sciences: An International Journal (AMS) Vol 8, No.1/2/3, August 2021

to raising the standards of hygiene within the hospital premises. Further, the model used gadgets like smart wheelchairs and body stretchers.

\section{Methodology}

\section{A. Experimental Planning and Preparation of Materials}

In this study, an experimental research design is used based on a recorded data that includes heart rate, blood pressure, oxygen levels, and body temperature gotten from the child subject seeking medical attention. The recorded body parameters are sent to the BalenaFin industrial grade development board via Bluetooth Low Energy (BLE) protocol. The board has Bluetooth, WIFI, and ethernet communication features.

The central development board referred to as the central device, has a program developed using python programming language, to hold and save the vitals, in this case, heart rate, body temperature, oxygen levels, and blood pressure.

The peripheral device is enabled to be easily discovered and connected to the central device using a MAC address. The actual MAC address of the device is also saved with the data of the child to assist identify the child and the age.

The central device operates in active mode since it is receiving the recorded data. The pairing process is

preconfigured and connections established with the data. Data received on the central device is first stored locally on a .csv file as backup, at the same time it is sent to a cloud server via the internet connection to the internet hotspot. The MQTT that transports messages between devices sends data to the cloud server and stores it in a database.

A cloud server is set up on an Amazon Web Service (AWS) instance with the Debian/Ubuntu operating system. Anaconda development environment installed on the virtual machine (cloud server) to facilitate the use of Jupyter notebook computational software. A NoSQL time series influx database is also installed for data storage from subscribed MQTT topics.

\section{B. Population, Sampling and Sample Size}

The study population included children seeking treatment at Murang'a level 5 hospital and Kangema level 4 hospital. Both hospitals are located in Murangacounty, Kenya. The two hospitals were chosen because they have specialized departments that deal with child healthcare. On average, 40 children visit Murang'a level 5 hospital while 30 children visit Kangema level 4 hospital per day. Thisdata was collected to assist in the general testing of the model. Therefore. We used purposive sampling to determine the sample size. The patient demographics were high since it served children from neighboring counties and referrals.

We used the following formula to calculate the sample size as proposed in Michael Slovin (1960)

$$
\mathrm{n}=\mathrm{N} /\left(1+\mathrm{Ne}^{\wedge} 2\right)
$$

Where $\quad n=$ number of sample size

$\mathrm{N}=$ Total population

$\mathrm{e}=$ confidential level. 
We got a confidence level of 0.02 . It provides a $98 \%$ level of accuracy. From a population of 40 at the Murang'a level 5 hospital, we obtained a sample size of 59 children i.e.

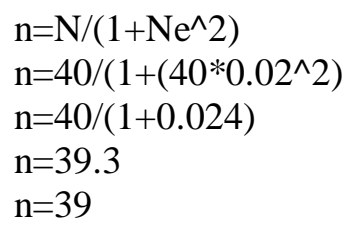

In addition, we obtained a sample of 33 children from a population of 33 at the Kangema level 4 hospital i.e.

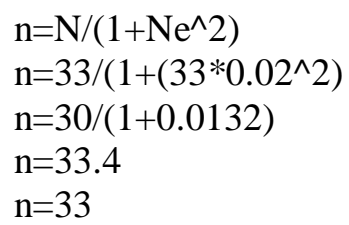

This information on sample sizes used in this study is shown in Table 1.

Table 1: Study Sample

\begin{tabular}{|l|l|l|}
\hline & Population & Sample \\
\hline $\begin{array}{l}\text { Murang'a } \\
\text { level 5 hospital }\end{array}$ & 40 & 39 \\
\hline $\begin{array}{l}\text { Kangema } \\
\text { level 4 hospital }\end{array}$ & 33 & 33 \\
\hline Total & 73 & 72 \\
\hline
\end{tabular}

\section{Data Collection}

In this study, Data recorded in the past three months in both hospitals was used to train the machine learning algorithm, that was implemented in the model.

\section{Data Analysis}

Heart rate, blood pressure, temperature, and oxygen levels were used to classify emergency cases. Random Forest classifier was used to make predictions on the data. Anaconda helps in analytics. It is a software that was installed along with numerous analytical tools and libraries including Jupyter notebook. A python3 virtual environment was set up and SciKit-Learn, scipy, NumPy, Matplotlib, pandas among other libraries. The virtual environment was created to keep all libraries native to the application and development. Using the Jupyter notebook a Random Forest Classifier is trained using hospital recorded data. $75 \%$ of the data was used in training while $25 \%$ validating the model accuracy. A large part of the dataset was used to train the model to achieve higher accuracy and only a small sample was used to validate.

\section{E. Ethical Considerations}

Issues such as children's privacy, data protection, accessibility, and rights were considered by ensuring that the data collected from the hospitals were only viewed by the specialists concerned. Also, in the creation of the model, only the medics were able to view the data on the frontend 
Advanced Medical Sciences: An International Journal (AMS) Vol 8, No.1/2/3, August 2021

screen. We also obtained permission from relevant local authorities and bodies to handle the data such as NACOSTI, county health services and the Ministry of education in Muranga, Kenya.

\section{IMPLEMENTATION}

\section{A. Factors Needed to Identify Children Emergency Cases}

Children show a specific range of symptoms to indicate a need for urgent medication. These symptoms are detected by the use of IoT - based gadgets since it is not easy to measure them accurately using standard equipment and also due to age. They include blood pressure, heart rate, body temperature, respiratory rate, and oxygen concentration.

Heart Rate: The heart rate indicates the number of times the heart beats per minute[7]. The child heart rate values are indicated in the heart rate table [8].

Body Temperature:

Body temperature in children is considered as one of the most contributing factors to medical consultation in children and referred to fever as a symptom that contributes up to $25 \%$ as to why consultation is crucial for children. [9]. From the research temperature can be as low as 360C when a child is asleep to $37.80 \mathrm{C}$ when the child is active, however for clinical and research purposes, fever is defined as 380c or higher. The most considered value for normal temperature for children is $36.5-37.50 \mathrm{C}$.

Blood Pressure: For children, blood pressure value depends on age. Children from birth to one month have a blood pressure value of 67 to 84 systolic blood pressure over 31 to 45 diastolic [10]. Children from one month to twelve months have a blood pressure of 72 to 104 systolic over 37 to 56 diastolic. When a child turns a year old, their blood pressure tends to progress towards adult values. For example, 1 to 2 years child has blood pressure values changes to systolic 86 to 106, diastolic 42 to 63 , a child aged 3 to 5 has a blood pressure of systolic 89 to 112, diastolic 46 to 72 , a child aged 6 to 11 years, have a blood pressure of systolic 97 to 120 , diastolic 57 to 80 . Twelve years and above have the same blood pressure as adults because their heart and breathing muscles have developed to almost the level of adult implying that their blood pressure is systolic 110 to 131 , diastolic 64 to 83

Oxygen levels: It is the amount of oxygen circulating in the blood. From the pediatric chart, the value ranges from $>90 \%$ to $100 \%$

\section{B. Health IOT - Based Gadget for Monitoring Children Health Cases.}

Pulse Oximeter:It is a device used to measure the oxygen level of the blood. It checks on how well oxygen is being sent to parts of your body furthest from your heart, such as the arms and legs.

Blood Pressure and Heart Rate Sensor: It can be explained as an IoT gadget, used to indicate the pressure of the blood. It uses a non-invasive method where the piercing is not necessary [11]. It measures systolic and diastolic, and it also indicates the values of the hate rate.

Temperature sensor: It is an IoT device used to determine the temperature level of a human being. [13]. 


\section{Model Design}

BalenaFin carrier board with Raspberry Pi compute module at its core, is the central device of the system. It is an industrial design grade, power-efficient, Bluetooth and Wi-Fi data transmission protocol enabled, and it is suitable for this model.

Both the recorded data and simulated data using python libraries, are separated in a way to clearly indicate the body temperature, heart rate, blood pressure, and oxygen concentration among others. The data is saved in a comma separated values file.

A Bluetooth network is designed with a piconet topology. The network has a central device, attached with a raspberry pi 3 module, and the data assumed to have been collected using peripheral devices from the hospitals. The central device is powered using jack barrel power supply type and connectivity is configured for both Bluetooth and Wi-Fi. A data packet Bluetooth scanning, collection, and saving python3 program is run on the central device.

Advertised data packets in this case the recorded and simulated data, are scanned, collected, and saved on a .csv file on the central device for backup awaiting transmission to a cloud server. The data is later sent to an AWS cloud server database via MQTT. It is from here, where the developed front-facing dash application query data, analyses using machine learning techniques and visualizes emergency inferences. Figure 1 shows the initial concept design. The following IoT technologies and devices were used;

\section{BalenaFin}

BalenaFin is a suitable industrial customized development board designed with the Raspberry Pi Compute Module 3 and 3+. It acts as a breakout and carrier board. It gives access to internal firmware (RasbianOs) and wireless communication protocols such as Bluetooth version 4.2 that comes on board as a radio connectivity protocol. It operates at a frequency of $2.4 \mathrm{GHZ}$, and can sense nearby Bluetooth devices up to a range of $10 \mathrm{~m}$.

It has a robust design for deployment in the field, for instance, in hospitals with suitable enclosures, and enough computational power for resource processes such as threading, pairing, connecting, and disconnecting. The board is powered by $6 \mathrm{~V}-24 \mathrm{~V}$ power sources. In addition, it has extended GPIO pins for scalability and integration with other pediatric systems. In this work, we took advantage of the computational resources, Bluetooth, and Wi-Fi wireless data transmission protocols onboarded.

The dual-band $802.11 \mathrm{ac}$ standard, $2.4 \mathrm{GHz}$ and also $5 \mathrm{GHz}$ Wi-Fi is also on the hardware and can simultaneously be used when in a place without a wired 10/100 Ethernet connection to send and receive data.

\section{Sensor's devices and simulated data}

Smart bands at times referred to as bracelets are modern generic wrist-worn electronic gadgets. They are mostly customized for health reference and sport activity monitoring. Initially, the devices were used as pedometers. However, the latest advancements have made the device applications increase significantly since 2012 (e.g in the health sector). They are cheaper and meet functionality, standard hardware, and software requirements. They can be bought off the shelf at a cost of less than Ksh 7000. Most of them are not accurate and need configuration and some information from the user such as age, weight, and height among others. 
Advanced Medical Sciences: An International Journal (AMS) Vol 8, No.1/2/3, August 2021

Accurate smart bracelets measure heart rate, blood pressure, oxygen levels, and body temperature when worn on the wrist. They have temperature, optical, ECG, photoelectric, and vibration sensors that can provide valuable information about a child. It is worn with a grip on the wrist for accurate results since the system is non-intrusive and can be affected by weather actors and surrounding environments.

Normally the bands have specific android applications to pair to the peripheral device and collect data. They have authentication keys that have expiry sessions per user.

\section{Cloud Server}

Data storage, data analysis, and serving the front-facing application are done on Amazon Web Services (AWS) ec2 cloud server. It is a t2-micro instance with Linux operating system. The data sent from the microcontroller is received in a database installed on the server. Also, the dash application developed was deployed on the instance. It has 8 vCPUs, and 64 GB of hard disc, providing enough memory to do basic computation and data storage.

\section{Software}

Development of the microcontroller firmware and algorithms was done in the python3 programming language. The Bluetooth data collection and transmission programs were also developed in python3 with the use of Bluetooth and Wi-Fi libraries and packages that help in channeling.

For data analysis and visualization, the Plotly Dash application was used since it is a productive python framework for building web analytic applications and has various data representation methods and charts. It was developed on top of Flask, React.js, and Plotly.js, which are ideal for building data visualization applications, with highly custom user interfaces in python. It was suited for data-related work in python. Data was queried from the database analyzed and visualized on the application.

\section{Data transmission protocols}

Data transmission protocols are set standards and sets of rules that allow computers and systems to exchange data. Also, they are responsible for how they interpret and format the data. Data transmission protocols are different depending on the need, application, and physical factors. It defines the packet structure and control commands that manage the communication session, for instance, Bluetooth wireless, IP(TC/IP), 802.11 wireless Wi-Fi, and ATM. Any application can employ one or more of these data transmission protocols. In this work, Wi-Fi, Ethernet and also Bluetooth were used. Ethernet was used for debugging, interfacing as well as sharing the internet with the central device. Bluetooth was used as an assumption that the recorded and simulated data, were transmitted to the central device using it

\section{Plotly Dash}

A dash application is a data analytical app purposely developed for this work to visualize and persist data to the pediatrician. It was developed on top of the flask, plotly.js, react.js framework, and python programming language. The application visualizes real-time data streams and handles real-time classification. In addition, data can be back-traced to view historical records and classify specific subsets of the data. 


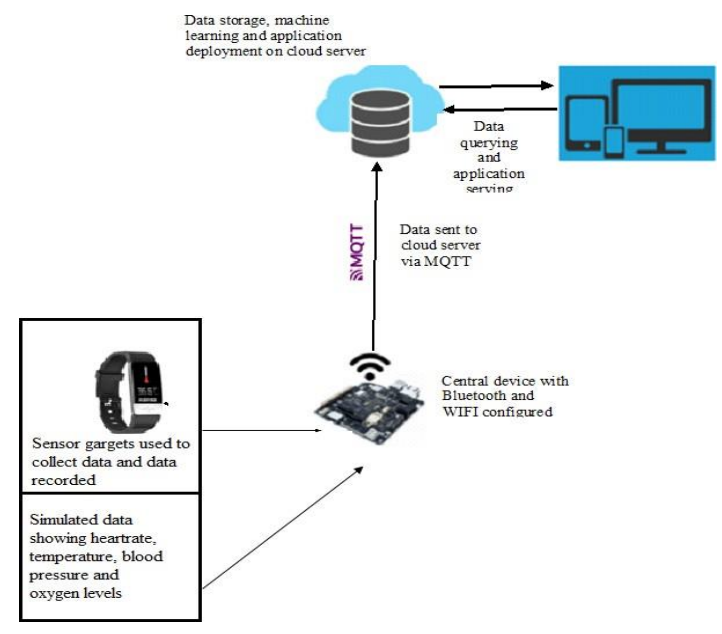

Figure 1: Initial conceptual diagram

\section{The central device, simulated and recorded data interoperability}

The operating system was installed on the compute module, a Raspbian Os. Wi-Fi name and password are configured to connect the device to the internet. The work of the central device is to act as a client from a client-server perspective by scanning and collecting simulated data and recorded advertised by python libraries and sensors gargets respectively. MAC addresses representing sensor garget are used whenever communication is needed. BalenaFin is powered with the barrel jack power supply and connected on Ethernet LAN cable to the computer via Secure Shell (SSH) for debugging and programming.

\section{Parameter Simulation, Data Collection, and Transmission to AWS}

A coding program to simulate data from peripheral devices was developed to generate and send data to the AWS cloud server for saving. The parameter ranges were selected carefully according to Murang'a Provincial Hospital and World Health Organization (WHO) standards for children's health for pediatric use.

The data was published to a health_channel topic and the server-side program got the data from the central device remotely. At the same time, the data is saved on the central device on a .csv file as shown below.

On the AWS IoT Core, the subscription program subscribes to the health_channel and saves the data to the influx time-series database. Timestamps for the recorded data are also recorded. The data saved in the influx database is as shown in figure 2 .

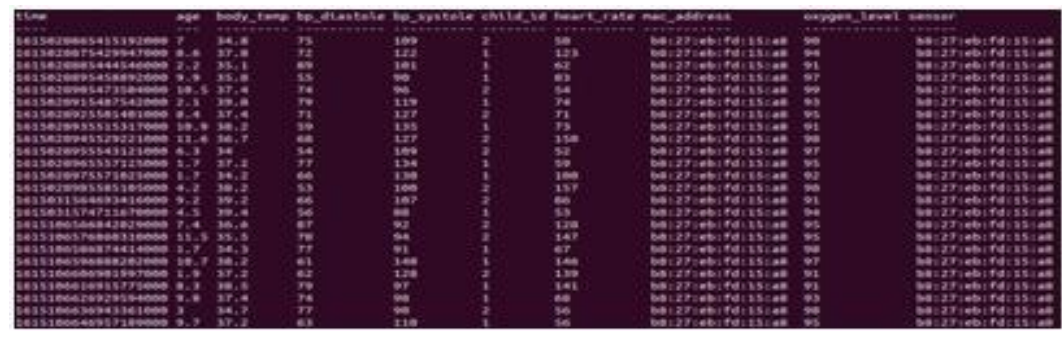

Figure 2: Simulated data saved in influxdb 
Advanced Medical Sciences: An International Journal (AMS) Vol 8, No.1/2/3, August 2021

Data collected were analyzed to uncover insights. A Random Forest classifier was trained using labeled data collected from the hospital records of about 820 records. Labeling entailed working closely with a pediatrician to uncover the emergency state based on the 6 parameters, namely, age, body temperature, blood pressure, heart rate, and oxygen level. 72 data points saved on a .csv file were used for the machine learning process.

There are three classes a child is classified into based on their vital body parameters. Important Sklearn modules are imported and data loaded to pandas data frame tables using pandas to create a data set for machine learning. The data is inspected and cleaned. Independent and dependent variables are declared using NumPy and later split into training and testing sets in the ratio of 3:1. Feature scaling is later done and the data fit the training set. The model is tested using the testing data set and the classifier feature importance analyzed as shown in Figure 3 to know the contribution and essence of each parameter in the training process.

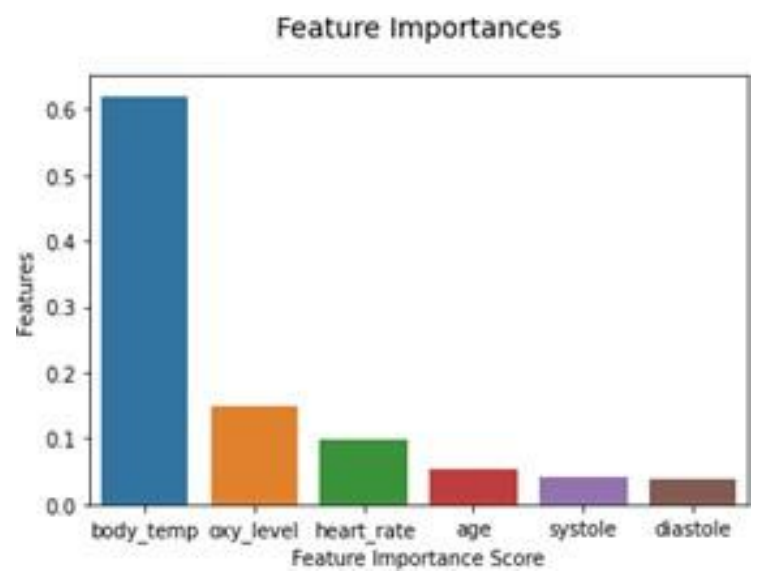

Figure 3: Feature importance

\section{Dash Application}

The glance view of all parameters on the dash application is as shown in Figure 4 where real-time data per child is visualized. Figure 5 shows the emergency classifications and top 10 latest children with their respective data records using the simulated data.

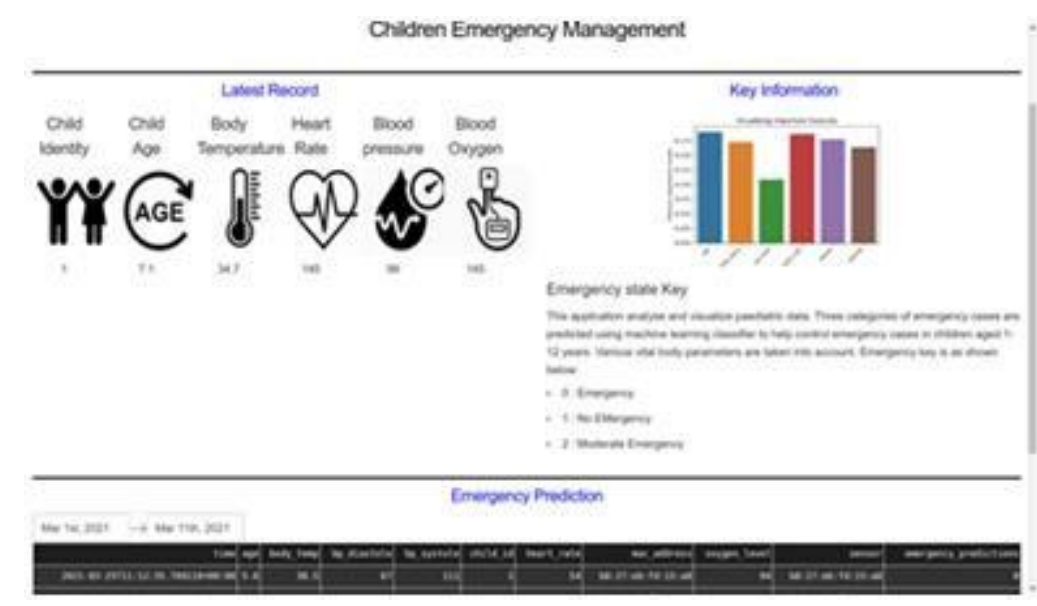

Figure 4: Parameter values visualization per child 


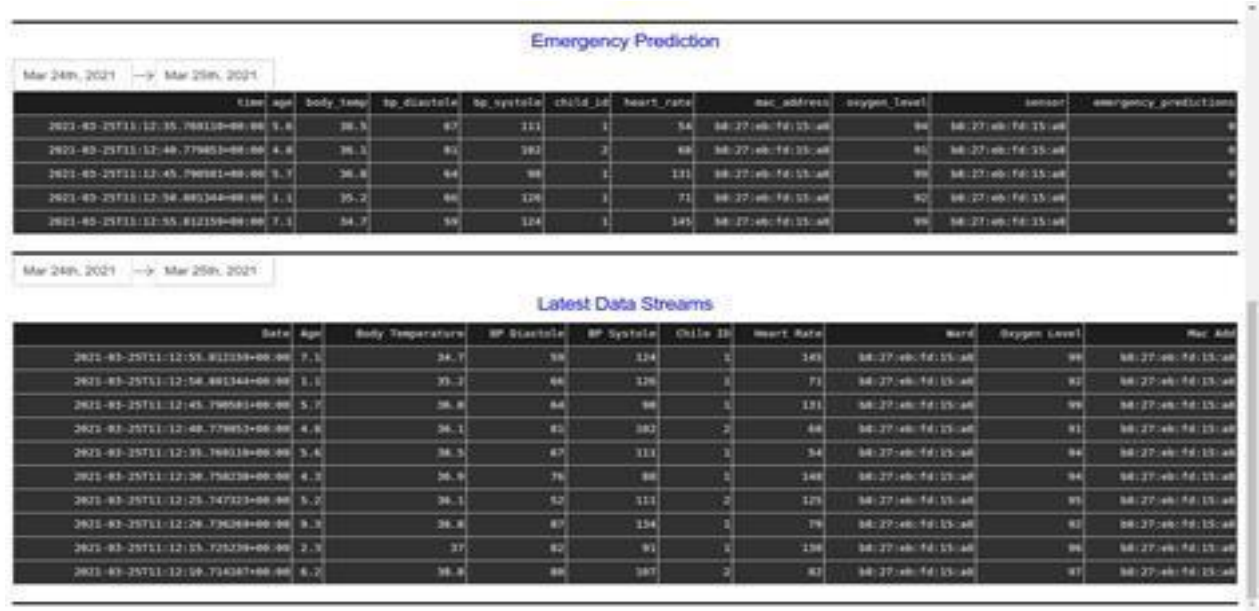

Figure 5: Plotly dash web application processing and visualizing data with emergency classes as inferences.

The Dash application ran in near real-time with auto-refresh. Any new data saved in the database is analyzed in real-time and classified as either emergency (class 0 ), no emergency (class 1), and moderate emergency (class 2). The children's IDs alongside their emergency classes are tabulated on a table for medics to plan the emergency cases. The trained model used Body temperature, oxygen concentration, heart rate, age, systole, and diastole as features. The three classes represent the emergency levels.

Top to latest children patients attending at that instance is displayed in real-time with functionality to retrieve historical children data. Body temperature and heart rate were the most essential features as seen in the feature importance score. The application is responsive on all platforms including mobile phones.

\section{RESUlts}

After the analysis of the data collected from the hospital records, it indicated that there were 36 emergency cases, 21 no emergency cases, and 15 moderate cases. The above analysis was done with the assistance of medics, wheretemperature was given the first priory, followed by oxygen levels and heartrate, and lastly the blood pressure. Blood pressure was given the least importance in the analysis because, rarely do children suffer from blood pressure related conditions.

In relation to both expert views and pediatric chart values, the table 2 below, indicates that the model displayed the following number of records after it was tested with the testing data from the hospitals.

Table 2: results of the model after using the testing data

\begin{tabular}{|c|c|c|}
\hline \multirow[t]{2}{*}{$\begin{array}{l}\text { Emergency } \\
\text { cases }\end{array}$} & $\begin{array}{l}\text { No. of records according to Expert Judgement / } \\
\text { pediatric chart }\end{array}$ & 36 \\
\hline & No. of records displayed by the model & 34 \\
\hline \multirow[t]{2}{*}{ Moderate cases } & $\begin{array}{l}\text { No. of records according to Expert Judgement / } \\
\text { pediatric chart }\end{array}$ & 15 \\
\hline & No. of records displayed by the model & 17 \\
\hline \multirow[t]{2}{*}{$\begin{array}{l}\text { Non- } \\
\text { emergency }\end{array}$} & $\begin{array}{l}\text { No. of records according to Expert Judgement / } \\
\text { pediatric chart }\end{array}$ & 21 \\
\hline & No. of records displayed by the model & 21 \\
\hline
\end{tabular}


Advanced Medical Sciences: An International Journal (AMS) Vol 8, No.1/2/3, August 2021

The confusion matrix in table 3 below shows the performance of the machine learning algorithm, its accuracy value being 0.968609865470852 , which is 0.97 in two decimal places, or $97 \%$ accuracy level

Table 3: Confusion matrix for the model

\begin{tabular}{|l|l|l|l|l|l|}
\hline 0.968609865470852 & & 0.97 & 223 & \\
\hline Accuracy & & & 0.96 & 223 & \\
\hline Macro avg & 0.96 & 0.96 & 0.97 & 223 & \\
\hline Weighted avg & 0.97 & 0.97 & \multicolumn{5}{l|}{} \\
\hline \multicolumn{5}{|l|}{} \\
\hline Predicted emergency status & EMERGENCY & MODERATE & NON EMERGENCY \\
\hline Actual Emergency Status & & \multicolumn{3}{l|}{} \\
\hline EMERGENCY & 46 & 4 & 0 \\
\hline MODERATE & 1 & 46 & 1 \\
\hline NON EMERGENCY & 0 & 1 & 124 \\
\hline
\end{tabular}

The trained algorithm was saved and used in a dash application used to classify the children's emergency status.

\section{DisCuSSION}

In a collection of 72 records for testing the model, the model was in a position to produce $100 \%$ accuracy, in displaying the exact records of moderate status children, which is a total of 21 out of 21 records, 94\% accuracy in displaying the exact records of emergencystatus children, which is a total of 34 out of 36 records, and it was able to display the exact records of moderate data that is 15 out of 15 records, though the two emergency status records were classified under moderate.

This can be improved by increasing the number of datasets when training the model and would result into a more reliable model to assist in pediatric section. This is mainly for arranging the pediatric children whose emergency status, cannot be viewed physically.

Several machine learning algorithms such as SVM, Decision tress and random forest classifiers were used to determine the best to be used in the model implementation, but the most effective one, was the random forest classifier since it was able to deal with vitals that do not have a big feature importance in the classification of children such as the blood pressure

\section{CONClusion ANd Future Work}

The model had a 97\% accuracy level, after it was tested with 72 records from a hospital where out of 21 non-emergency records, it displayed all the 21 records, out of 36 emergency records, it displayed 34 records and out of 15 moderate, it added two emergency records to the group.

To further this work, more data should be used to further train the model, and raise the accuracy to $100 \%$. Also, Exploration of Bluetooth range and other architecture should be done to open new ways of covering other age groups and utilize other body parameters. In addition, the implementation of an alert system on this work is vital to alert pediatricians when attending to 
Advanced Medical Sciences: An International Journal (AMS) Vol 8, No.1/2/3, August 2021

multiple patients. Hence future work should focus on alert systems. Time spent with each patient can also be explored to unfold the quality of service intelligence from the data collected.

\section{REFERENCES}

[1] P. McCafferty, "Implementing Article 12 of the United Nations Convention on the Rights of the Child in Child Protection Decision-Making: a Critical Analysis of the Challenges and Opportunities for Social Work", Jun. 09, 2021.

[2] P. Svedberg, S. Arvidsson, I. Larsson, I.-M. Carlsson, and J. M. Nygren, "Barriers and Enablers Affecting Successful Implementation of the Electronic Health Service Sisom: Multicenter Study of Child Participation in Pediatric Care," J. Med. Internet Res., vol. 21, no. 11, p. e14271, Nov. 2019.

[3] W. Jin and D. H. Kim, "Design and Implementation of e-Health System Based on Semantic Sensor Network Using IETF YANG,” Sensors, vol. 18, no. 2, Feb. 2018, DOI: 10.3390/s18020629.

[4] A. Kakkar and A. Garg, "Pervasive Health Monitoring of Special Child using IoT and Cloud Technologies," 2019 International Conference on Intelligent Computing and Control Systems (ICCS). 2019, DOI: 10.1109/iccs45141.2019.9065375.

[5] P. Jangra and M. Gupta, "A Design of Real-Time Multilayered Smart Healthcare Monitoring Framework Using IoT,” 2018 International Conference on Intelligent and Advanced System (ICIAS). 2018, DOI: 10.1109/icias.2018.8540606.

[6] N. Petrellis, M. Birbas, and F. Gioulekas, "On the Design of Low-Cost IoT Sensor Node for e-Health Environments," Electronics, vol. 8, no. 2. p. 178, 2019, DOI: 10.3390/electronics8020178.

[7] S. Fleming et al., "Normal ranges of heart rate and respiratory rate in children from birth to 18 years of age: a systematic review of observational studies," Lancet, vol. 377, no. 9770, pp. 1011-1018, Mar. 2011.

[8] C. Novak, K. Forbes, and P. Gill, "PedsCases: A Learning Module for the Approach to Pediatric Anemia," MedEdPORTAL, vol. 12, no. 1. 2016, DOI: 10.15766/mep_2374-8265.10319.

[9] E. Barbi, P. Marzuillo, E. Neri, S. Naviglio, and B. S. Krauss, "Fever in Children: Pearls and Pitfalls," Children, vol. 4, no. 9, Sep. 2017, DOI: 10.3390/children4090081.

[10] J. T. Flynn et al., "Clinical Practice Guideline for Screening and Management of High Blood Pressure in Children and Adolescents," Pediatrics, vol. 140, no. 3. p. e20171904, 2017, DOI: 10.1542/peds.2017-1904.

[11] M. Pasha and S. M. W. Shah, "Framework for E-Health Systems in IoT-Based Environments," Wireless Communications and Mobile Computing, vol. 2018. pp. 1-11, 2018, DOI: $10.1155 / 2018 / 6183732$.

[12] M. Sharma, S. Kacker, and M. Sharma, "A Brief Introduction and Review on Galvanic Skin Response," International Journal of Medical Research Professionals, vol. 2, no. 6. 2016, DOI: 10.21276/ijmrp.2016.2.6.003.

[13] Z. Alansari, S. Soomro, M. R. Belgaum, and S. Shamshirband, "The Rise of Internet of Things (IoT) in Big Healthcare Data: Review and Open Research Issues," Advances in Intelligent Systems and Computing. pp. 675-685, 2018, DOI: 10.1007/978-981-10-6875-1_66. 